\title{
Prolonged Time from Diagnosis to Breast-Conserving Surgery is Associated with Upstaging in Hormone Receptor-Positive Invasive Ductal Breast Carcinoma
}

\author{
Natalie Hills, BS ${ }^{1}$, Macall Leslie, BS$^{2}$, Rachel Davis, BS$^{1}$, Marielle Crowell, BS $^{1}$, Hiroyasu Kameyama, PhD $^{2}$, \\ Hallgeir Rui, $\mathrm{MD}, \mathrm{PhD}^{3}$, Inna Chervoneva, $\mathrm{PhD}^{4}$, William Dooley, $\mathrm{MD}^{5}$, and Takemi Tanaka, $\mathrm{PhD}^{2,6}$ \\ ${ }^{1}$ University of Oklahoma Health Sciences Center, College of Medicine, Oklahoma City, OK; ${ }^{2}$ University of Oklahoma \\ Health Sciences Center, College of Medicine, Stephenson Cancer Center, Oklahoma City, OK; ${ }^{3}$ Department of Pathology, \\ Medical College of Wisconsin, Milwaukee, WI; ${ }^{4}$ Division of Biostatistics, Department of Pharmacology and Experimental \\ Therapeutics, Thomas Jefferson University, Philadelphia, PA; ${ }^{5}$ Department of Surgery, University of Oklahoma Health \\ Sciences Center, College of Medicine, Oklahoma City, OK; ${ }^{6}$ Department of Pathology, University of Oklahoma Health \\ Sciences Center, College of Medicine, Oklahoma City, OK
}

\begin{abstract}
Background. Time to surgery (TTS) has been suggested to have an association with mortality in early-stage breast cancer.

Objective. This study aims to determine the association between TTS and preoperative disease progression in tumor size or nodal status among women diagnosed with clinical T1N0M0 ductal breast cancer.

Methods. Women diagnosed with clinical T1N0M0 ductal breast cancer who had breast-conserving surgery as their first definitive treatment between 2010 and 2016 $(n=90,405)$ were analyzed using the National Cancer Database. Separate multivariable logistic regression
\end{abstract}

Natalie Hills, Macall Leslie, and Rachel Davis have contributed equally to this work.

Disclaimer The NCDB is a joint project of the CoC of the American College of Surgeons and the American Cancer Society. The CoC's NCDB and the hospitals participating in the CoC's NCDB are the source of the de-identified data used herein; they have not verified and are not responsible for the statistical validity of the data analysis or the conclusions derived by the authors.

(C) The Author(s) 2021

First Received: 23 October 2020

Accepted: 6 January 2021;

Published Online: 21 March 2021

T. Tanaka, $\mathrm{PhD}$

e-mail: takemi-tanaka@ouhsc.edu models for hormone receptor (HR)-positive and HR-negative patients, adjusted for clinical and demographic variables, were used to assess the relationship between TTS and upstaging of tumor size (T-upstaging) or nodal status (N-upstaging).

Results. T-upstaging occurred in $6.76 \%$ of HR-positive patients and $11.00 \%$ of HR-negative patients, while $\mathrm{N}$-upstaging occurred in $12.69 \%$ and $10.75 \%$ of HR-positive and HR-negative patients, respectively. Among HRpositive patients, odds of T-upstaging were higher for 61-90 days TTS (odds ratio [OR] 1.18, 95\% confidence interval $[\mathrm{CI}] 1.05-1.34$ ) and $\geq 91$ days TTS (OR 1.47, 95\% CI 1.17-1.84) compared with $\leq 30$ days TTS, and odds of $\mathrm{N}$ - upstaging were higher for $\geq 91$ days TTS (OR 1.35, 95\% CI 1.13-1.62). No association between TTS and either $\mathrm{T}$ - or N-upstaging was found among HR-negative patients. Other clinical and demographic variables, including grade, tumor location, and race/ethnicity, were associated with both $\mathrm{T}$ - and $\mathrm{N}$-upstaging.

Conclusion. TTS $\geq 61$ and $\geq 91$ days was a significant predictor of T- and N-upstaging, respectively, in HR-positive patients; however, TTS was not associated with upstaging in HR-negative breast cancer. Delays in surgery may contribute to measurable disease progression in T1N0M0 ductal breast cancer.

Breast cancer is one of the most commonly diagnosed malignancies in the U.S. ${ }^{1,2}$ Staging at the time of diagnosis is an independent prognostic factor in breast cancer, ${ }^{3}$ and 
survival rates steeply decline with increasing stage. ${ }^{4}$ Clinical stage is determined by radiographic measurement of lesion size and disease spread to regional lymph nodes and distant organs. ${ }^{5}$ Subsequent pathologic staging is based on pathologic evaluation of tumor size and lymph node involvement in surgically resected lesions, along with the presence or absence of distant metastasis. Accordingly, upstaging is defined as a progressive discordance of tumor size, lymph node involvement, or distant metastasis between clinical and pathologic staging. Current literature suggests that dense breast tissue, palpable tumors, high grade, pleomorphic calcifications, lymphovascular invasion, and use of core-needle rather than vacuum-assisted biopsy are factors significantly associated with upstaging of ductal carcinoma in situ (DCIS) to invasive ductal carcinoma (IDC). ${ }^{6-8}$ A recent study suggested that prolonged time from diagnosis to surgery (time to surgery [TTS]) $\geq 30$ days is also associated with increased risk of upstaging from DCIS to IDC (median 38 days, range 30-365 days). ${ }^{8}$ Additional studies have demonstrated delay in surgery in early-stage breast cancer is associated with increased mortality risk, ${ }^{9,10}$ raising the question of whether TTS is also associated with measurable disease progression; however, previous studies examining the association between TTS and disease progression of invasive disease have not found a link. ${ }^{11-13}$ This study aims to analyze the association between upstaging and TTS by hormone receptor (HR) status among women diagnosed with clinical T1N0M0 ductal breast cancer who received breast-conserving surgery (BCS) as their first treatment. We also analyzed other clinical and sociodemographic factors associated with upstaging.

\section{METHODS}

\section{Cohort}

Women with clinical T1N0M0 ductal breast cancer diagnosed by needle or incisional biopsy between 2010 and 2016 who received BCS as their first definitive treatment were selected from the National Cancer Database (NCDB). The NCDB is a joint project of the Commission on Cancer $(\mathrm{CoC})$ of the American College of Surgeons and the American Cancer Society, and all data used in this study were de-identified and met the criteria for exempt review by the University of Oklahoma Health Sciences Center Institutional Review Board (IRB\# 7446). Women were selected based on a clinical stage of T1N0M0, ${ }^{14}$ ductal histology (International Classification of Diseases for Oncology, Third Revision [ICD-O-3] codes 8022, 8035, $8500-8503,8523)$, and receipt of BCS as their first treatment (Surgery of the Primary Site codes 19-24). Only cases diagnosed and treated, all or in part, at the reporting facility were included in the final cohort. Cases with previous, concurrent, or subsequent malignancies, missing clinical or pathologic staging, no biopsy or missing biopsy information, neoadjuvant treatment, non-definitive surgery (i.e. excisional biopsy) as their first treatment, or with incomplete covariate data, were excluded (Fig. 1). Patients with pathologic stage IV disease were excluded from analyses due to the likelihood of undetected metastases present at the time of diagnosis rather than new clinically detectable metastases developing within the preoperative timeframe ( $n=36,0.03 \%$ ). Additionally, the number of patients downstaged to pathologic in situ only tumors was insufficient ( $n=47,0.04 \%$ ) to be examined as a separate outcome and was excluded from the analyses. Finally, patients who did not have any nodes examined or who had an unknown number examined were excluded due to discordance with the National Comprehensive Cancer Network/American Joint Committee on Cancer guidelines for determining pathologic stage. ${ }^{14,15}$

Exclusion Scheme for Study Cohort

\begin{tabular}{|c|c|}
\hline \multicolumn{2}{|c|}{$\begin{array}{l}\text { Women diagnosed with breast cancer } \\
\text { NCDB, 2010-2016 (n=1,569,607) }\end{array}$} \\
\hline \multicolumn{2}{|c|}{ Breast cancer 1 st and only cancer $(n=1,184,867)$} \\
\hline \multicolumn{2}{|c|}{$\begin{array}{l}\text { Exclusions: } \\
\text { - Neoadjuvant treatment, non-definitive } \\
\text { surgery, no or missing biopsy information, } \\
\text { TTS } \leq 7 \text { or }>180 \text { days }(\mathrm{n}=498,472) \\
\text { - } \quad \text { Not diagnosed or treated at least partially } \\
\text { at reporting facility }(\mathrm{n}=355,578) \\
\text { - } \text { Clinical Stage } 0 \text { or II-IV, node positive, non } \\
\text {-IDC histology, pathologically DCIS or } \\
\text { metastatic }(\mathrm{n}=202,440) \\
\text { - } \\
\text { Mastectomy and other non-breast } \\
\text { conserving treatment }(\mathrm{n}=30,384) \\
\text { Missing covariate data }(\mathrm{n}=7,588)\end{array}$} \\
\hline $\begin{array}{c}\text { HR-positive } \\
\text { patients } \\
(\mathrm{ER} \text { and/or } \mathrm{PR}+) \\
\mathrm{n}=81,122\end{array}$ & $\begin{array}{l}\text { HR-negative } \\
\text { Patients } \\
\text { (ER-/PR -) } \\
\text { n=9,283 }\end{array}$ \\
\hline
\end{tabular}

FIG. 1 Exclusion scheme. $N C D B$ National Cancer Database, TTS time to surgery, IDC invasive ductal carcinoma, DCIS ductal carcinoma in situ, $H R$ hormone receptor, $E R$ estrogen receptor, $P R$ progesterone receptor 


\section{Exposure}

TTS was defined as the number of days between definitive diagnosis by biopsy and surgery and categorized by monthly intervals (i.e. $\leq 30,31-60,61-90$, and $\geq 91$ days). Patients who received surgery in 7 or fewer days were excluded from our study since receipt of surgery within 1 week of definitive diagnosis is unlikely in the modern setting ( $n=1,824,1.97 \%)$ and may be clinically reflective of greater medical urgency or unique circumstances. Additionally, patients with TTS over 6 months (180 days) were excluded from the study cohort due to exceptional delay $(n=127,0.14 \%)$.

\section{Outcome}

Upstaging was defined by two separate measures: $\mathrm{T}$-upstaging as an increase in tumor size from clinical $\mathrm{T} 1$ to pathologic $\mathrm{T} 2$ or higher, and $\mathrm{N}$-upstaging as a change in nodal status from clinical N0 to pathologic N1 or higher.

\section{Definitions}

The cohort was grouped into two categories according to HR status (i.e. estrogen and/or progesterone)—HR-positive or HR-negative. HER2 status was determined using collaborative staging site-specific factor, ${ }^{15,16}$ which summarizes the results of immunohistochemistry and geneamplification test (fluorescence in situ hybridization or chromogenic in situ hybridization) scoring of HER2 status as HER2-positive, HER2-borderline/equivocal, or HER2negative. Patient race/ethnicity was categorized as White, Black, Hispanic, or other (Asian, Pacific Islander, American Indian/Eskimo/Aleutian), and age was dichotomized by the average age of menopausal onset $(\leq 50$ or $>50$ years), since menopausal status at the individual level was unavailable in the NCDB. Number of nodes examined during surgery was categorized as $1,2-3,4-5$, or $\geq 6$. Location of tumor was classified according to ICD-O-3 codes as nipple/central portion (50.0, 50.1), upper inner quadrant (50.2), lower inner quadrant (50.3), upper outer quadrant (50.4), lower outer quadrant (50.5), axillary tail (50.6), or overlapping/other $(50.8,50.9)$.

\section{Statistics}

Patient characteristics were summarized by frequency and proportion for both $\mathrm{T}$ - and $\mathrm{N}$-upstaging. For continuous variables, the median, range, first quartile (Q1), and third quartile (Q3) were used to summarize their distributions. Separate binary multivariable logistic regression models were used to examine the association between TTS and the outcome of either $\mathrm{T}$ - or $\mathrm{N}$-upstaging, adjusted for dichotomized age, HER2 status, grade, tumor location, patient race/ethnicity, Charlson comorbidity score, and, for $\mathrm{N}$-upstaging models, number of nodes examined. All statistical analyses were conducted using SAS software version 9.4 (SAS Institute, Inc., Cary, NC, USA), and graphs were generated using JMP version 14 .

\section{RESULTS}

\section{Composition of the Cohort}

Following exclusions, the final cohort included 90,405 patients diagnosed with clinical T1N0M0 ductal carcinoma who received BCS between 2010 and 2016, with $89.73 \%$ ( $n=81,122)$ HR-positive and $10.26 \%(n=9,283) \mathrm{HR}$ negative. The median age at diagnosis was 64 years (range 23-90; Q1: 56; Q3: 71) among HR-positive patients and 62 years (range 24-90; Q1: 55; Q3: 70) among HR-negative patients. The median TTS was 28 days (Q1: 20; Q3: 40) among HR-positive patients and 27 days (Q1: 19; Q3: 38) among HR-negative patients, and the proportion of TTS was also similar in both groups ( $\leq 30$ days: $56.82 \% ; 31-60$ days: $36.88 \%$; $61-90$ days: $5.17 \%$; $\geq 91$ days: $1.13 \%$, in HR-positive patients; $\leq 30$ days: $60.09 \%$; 31-60 days: 34.31\%; 61-90 days: $4.61 \%$; $\geq 91$ days: $0.99 \%$, in HRnegative patients). The proportion of $\mathrm{T}$-upstaging grew with increasing TTS, from $6.6 \%$ of those with TTS $\leq 30$ days to $9.59 \%$ of those with TTS $\geq 91$ days among HRpositive patients; and from $10.77 \%$ of those with TTS $\leq 30$ days to $13.04 \%$ of those with TTS $\geq 91$ days among HRnegative patients (Table 1). Overall, 6.76\% $(n=5,483)$ of HR-positive patients and $11.00 \%(n=1,021)$ of HR-negative patients experienced upstaging from clinical $\mathrm{T} 1$ to pathologic T2 or higher (Table 1). The highest rates of T-upstaging in HR-positive patients were seen in tumors that were grade 3 or higher (13.99\%), HER2-positive $(9.80 \%)$, or located in the nipple/central portion of the breast $(8.99 \%)$ and among Hispanic patients $(9.06 \%)$. HRnegative patients who were $\leq 50$ years of age $(15.50 \%)$ or Hispanic (15.26\%), or who had a Charlson Comorbidity Index $\geq 2(13.32 \%)$ were more frequently T-upstaged (Table 1). With regard to $\mathrm{N}$-upstaging, $12.69 \%$ ( $n=10,298)$ of HR-positive cases and 10.75\% $(n=998)$ of HR-negative cases were upstaged from clinical NO to pathologic N1 or higher. The proportion of patients $\mathrm{N}$-upstaged also grew with increasing intervals of TTS, i.e. from $12.69 \%$ of HR-positive patients with $\leq 30$ days TTS to $16.34 \%$ of patients with $\geq 91$ days TTS upstaged, and from $10.81 \%$ of HR-negative patients with $\leq 30$ days TTS to $16.30 \%$ of patients with $\geq 91$ days TTS. N-upstaging was most prevalent in HR-positive cases that were HER2positive $(14.83 \%)$, located in the axillary tail $(18.06 \%)$ or 


\begin{tabular}{|c|c|c|c|c|c|c|c|c|c|c|}
\hline & 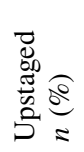 & $\begin{array}{l}\stackrel{0}{n} \\
\stackrel{0}{\sigma} \\
\infty \\
\sigma\end{array}$ & 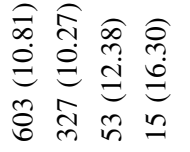 & 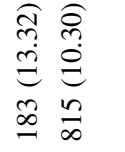 & 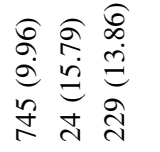 & 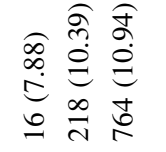 & 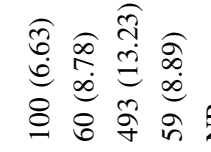 & & 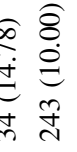 & 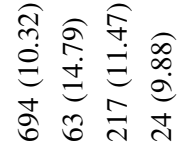 \\
\hline 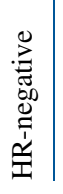 & 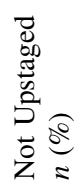 & 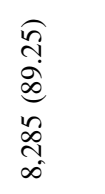 & 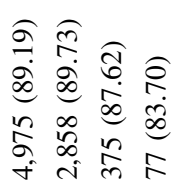 & 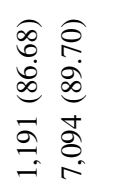 & 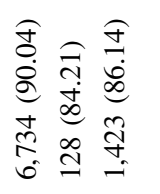 & 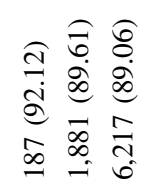 & 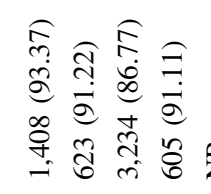 & & 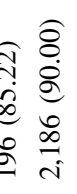 & 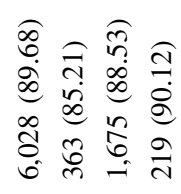 \\
\hline & 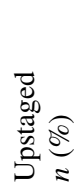 & $\begin{array}{l}\widehat{\hat{\sigma}} \\
\text { id } \\
\infty \\
o \\
\hat{i} \\
\stackrel{0}{0}\end{array}$ & 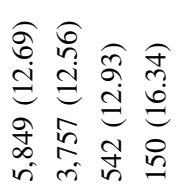 & 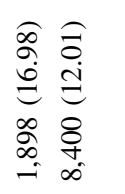 & 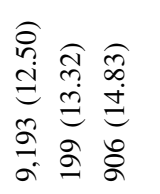 & 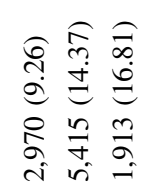 & 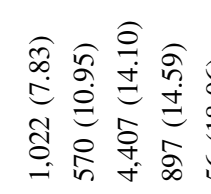 & & 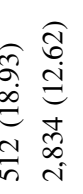 & 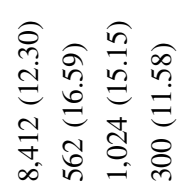 \\
\hline 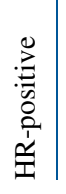 & 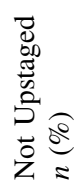 & 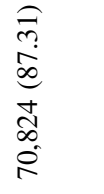 & 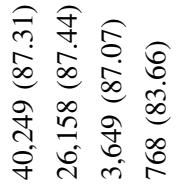 & 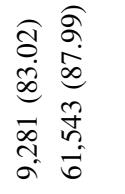 & 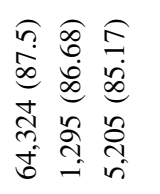 & 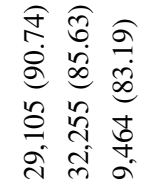 & 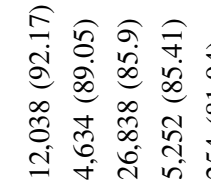 & & 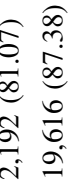 & 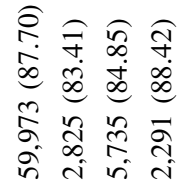 \\
\hline & 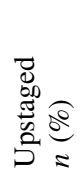 & 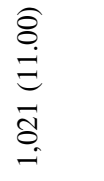 & 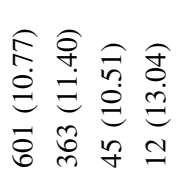 & 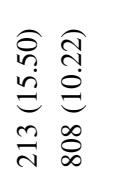 & 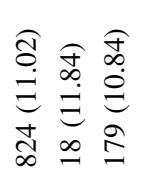 & 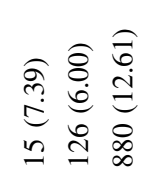 & 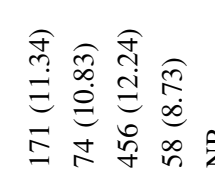 & & 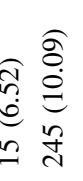 & 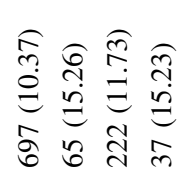 \\
\hline 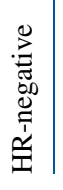 & 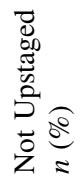 & 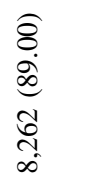 & 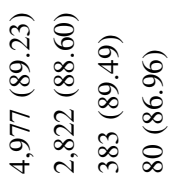 & 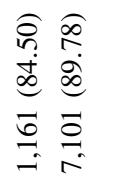 & 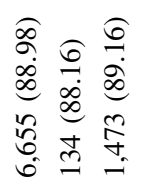 & 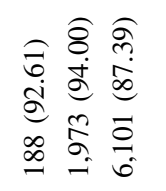 & 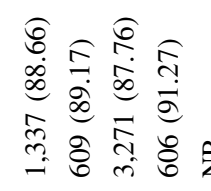 & & 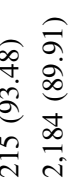 & 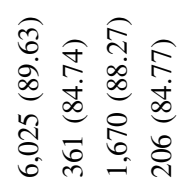 \\
\hline & 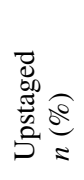 & 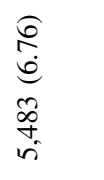 & 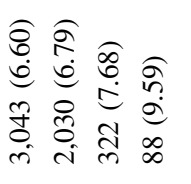 & 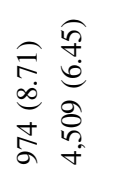 & 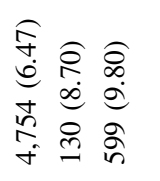 & 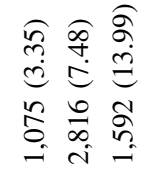 & 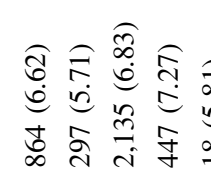 & & 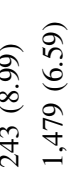 & 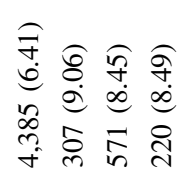 \\
\hline 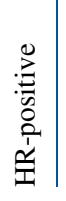 & 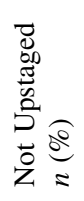 & 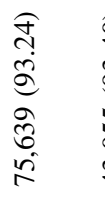 & 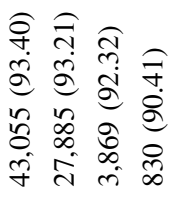 & 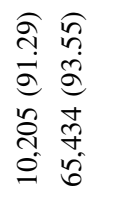 & 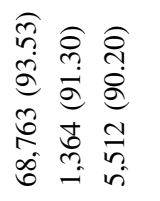 & 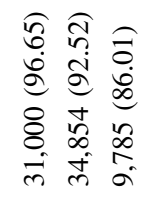 & 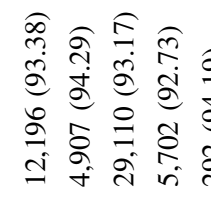 & & 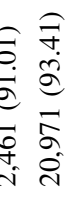 & 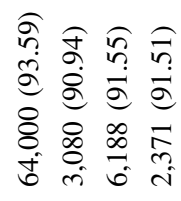 \\
\hline & & 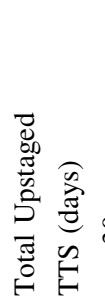 & 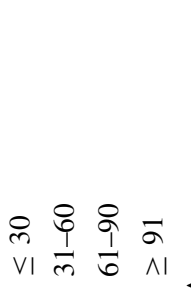 & & 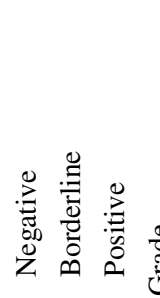 & $\wedge$ & 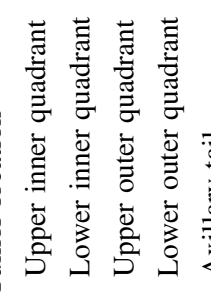 & & 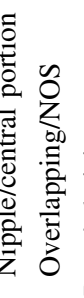 & 5 \\
\hline
\end{tabular}




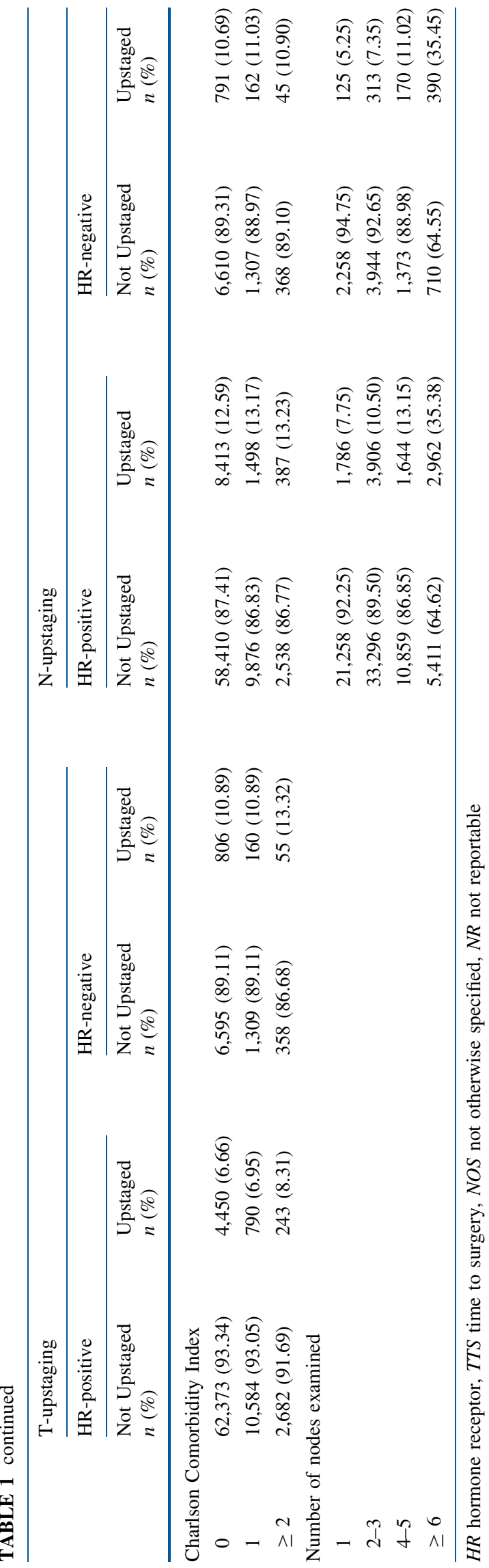

nipple/central portion of the breast $(18.93 \%)$, or had $\geq 6$ nodes examined (35.38\%), as well as among those who were Hispanic (16.59\%), Black (15.15\%), or $\leq 50$ years of age $(16.98 \%)$ [Table 1]. Higher proportions of $\mathrm{N}$-upstaging occurred among HR-negative patients with tumors located in the nipple/central portion of the breast $(14.78 \%)$, had $\geq 6$ lymph nodes examined $(35.45 \%)$, or who were Hispanic $(14.79 \%)$ or $\leq 50$ years of age $(13.32 \%)$ [Table 1 ].

Time to Surgery is Associated with Both T- and NUpstaging in Hormone Receptor-Positive Patients

Adjusting for the clinical and sociodemographic characteristics detailed in Table 1, HR-positive patients experienced a statistically significant increase in T-upstaging among those with 61-90 days TTS (OR 1.18, 95\% CI 1.05-1.34) and $\geq 91$ days TTS (OR 1.47, 95\% CI 1.17-1.84) compared with those with $\leq 30$ days TTS, while those with 31-60 days TTS did not experience a significant increase in risk (OR 1.04, 95\% CI 0.98-1.1) [Fig. 2a]. However, TTS of any interval $>30$ days was not associated with T-upstaging in HR-negative patients after multivariable adjustment (Fig. 2b). Similarly, TTS was significantly associated with $\mathrm{N}$-upstaging at $\geq 91$ days TTS (OR 1.35, 95\% CI 1.12-1.62) compared with TTS $\leq 30$ days among HR-positive patients (Fig. 2c), but not at $31-60$ or $61-90$ days TTS. There was not a significant association between TTS and N-upstaging in the HR-negative model (Fig. 2d).

Other clinical and demographic factors were also associated with $\mathrm{T}$ - and $\mathrm{N}$-upstaging, with statistical significance and effect sizes different according to HR status. Higher histologic grade was significantly associated with T-upstaging (OR 2.31, 95\% CI 2.15-2.48 in grade 2; OR 4.58, 95\% CI 4.21-4.98 in grade 3 or higher) and $\mathrm{N}$-upstaging (OR 1.58, 95\% CI 1.50-1.66 in grade 2; OR 1.80, 95\% CI 1.68-1.92 in grade 3 or higher) in HR-positive patients, while among HR-negative patients, only grade 3 or higher was significantly associated with T-upstaging (OR 1.72, 95\% CI 1.01-2.93) [Table 2]. HER2 status was not significantly associated with T-upstaging, but was associated with higher odds of $\mathrm{N}$-upstaging among HR-negative patients (OR 1.63, 95\% CI 1.01-2.62 in HER2-borderline; OR $0.51,95 \%$ CI $1.27-1.78$ in HER2-positive) [Table 2]. HR-positive tumors located in the nipple/central portion of the breast had higher odds of T-upstaging (OR 1.28, 95\% CI 1.11-1.47) and N-upstaging (OR 1.41, 95\% CI 1.27-1.57) than those located in the upper outer quadrant [Table 2]. The likelihood of $\mathrm{N}$-upstaging grew substantially with increasing number of nodes examined. Patients who had $\geq 6 \mathrm{removed} /$ examined were more likely to be N-upstaged compared with those with only 1 node examined regardless of HR status (OR 6.17, 95\% CI 5.77-6.59 in 
FIG. 2 Association between TTS and T-upstaging (a, b) and $\mathrm{N}$-upstaging (c, d) in HRpositive (left panel) and HRnegative (right panel). OR and CI were calculated relative to the reference ( $\leq 30$ days TTS). TTS time to surgery, $H R$ hormone receptor, $O R$ odds ratio, $C I$ confidence interval
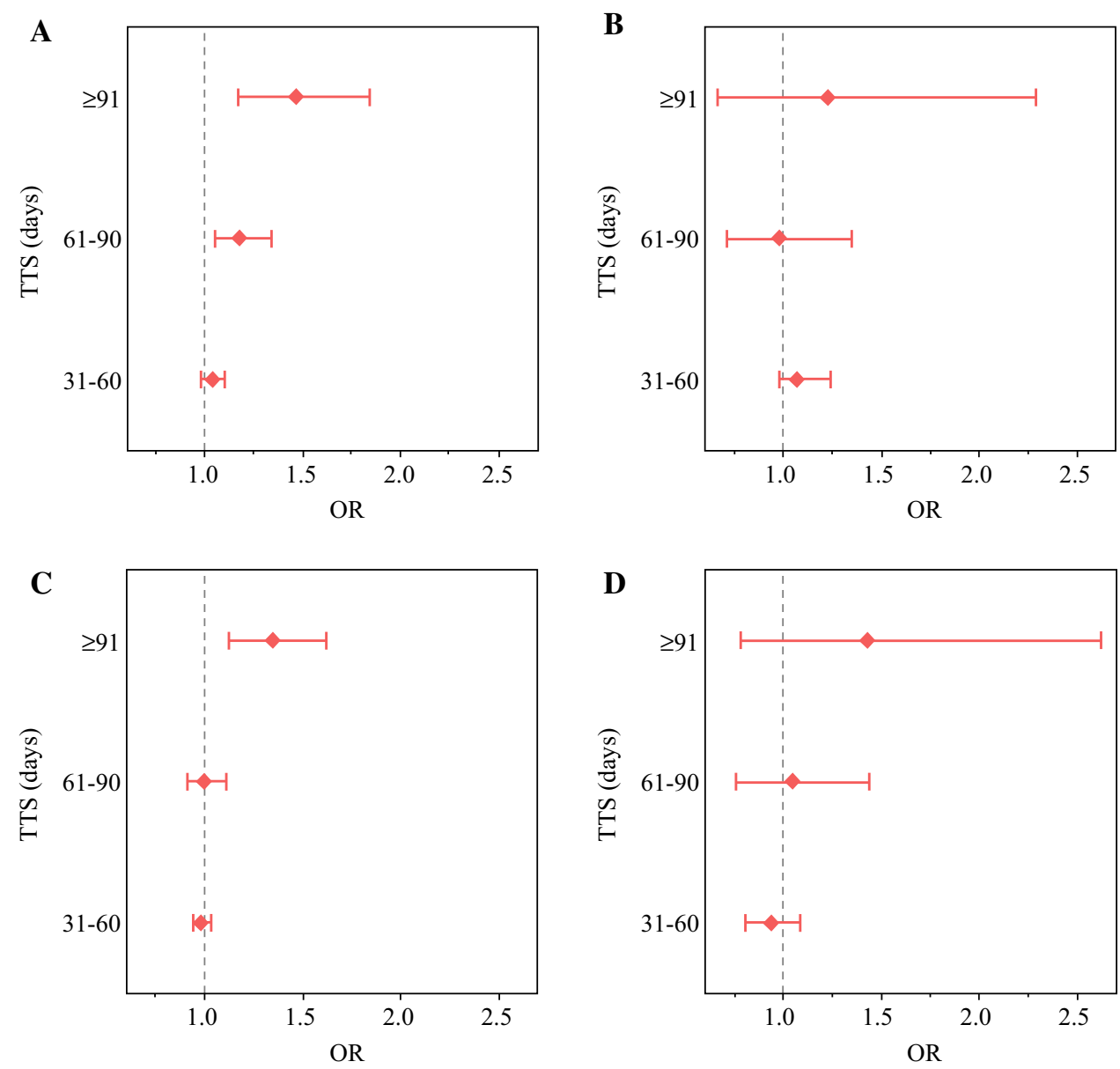

HR-positive patients; OR 9.6, 95\% CI 7.71-11.97 in HRnegative patients). In terms of demographic variables, patients $\leq 50$ years of age had statistically significant higher odds of T-upstaging (OR 1.18, 95\% CI 1.09-1.28 in HR-positive patients; OR 1.42, 95\% CI 1.18-1.72 in HRnegative patients) and $\mathrm{N}$-upstaging (OR 1.26, 95\% CI $1.18-1.34$ in HR-positive patients; OR 1.19 , 95\% CI $0.98-1.46$ in HR-negative patients), regardless of HR status. Additionally, HR-positive patients with a Charlson comorbidity index score of $\geq 2$ were significantly more likely to be T-upstaged (OR 1.28 , 95\% CI 1.11-1.46) or $\mathrm{N}$-upstaged (OR 1.13, 95\% CI 1.01-1.27) than those without comorbidities (Table 2). Finally, regardless of HR status, Hispanic patients experienced significantly higher odds of T-upstaging (OR 1.28, 95\% CI 1.13-1.45 in HRpositive patients; OR $1.40,95 \%$ CI $1.06-1.85$ in HR-negative patients) and $\mathrm{N}$-upstaging (OR 1.27, 95\% CI 1.15-1.40 in HR-positive patients; OR $1.38,95 \%$ CI $1.02-1.86$ in HR-negative patients). Similarly, Black HRpositive patients had higher odds of T-upstaging (OR 1.10, 95\% CI 1.00-1.12) and $\mathrm{N}$-upstaging (OR 1.10, 95\% CI 1.02-1.18) than White patients (Table 2).

\section{DISCUSSION}

Multivariable analyses of clinical T1N0M0 ductal breast carcinoma patients who received BCS as their first definitive treatment revealed that the preoperative period (TTS) was a significant predictor of disease progression in HRpositive patients, with $8 \%$ and $30 \%$ higher odds of T-upstaging at $\geq 61$ and $\geq 91$ days TTS, respectively, and $17 \%$ higher odds of N-upstaging at $\geq 91$ days TTS. Studies demonstrating a positive association between TTS and mortality risk among patients with early-stage breast cancer, ${ }^{9,10}$ as well as a positive association between TTS and upstaging of DCIS, ${ }^{8,13}$ raised the question of whether a prolonged preoperative interval is associated with measurable disease progression. However, earlier studies have not shown an association between TTS and upstaging in invasive disease. ${ }^{1-13}$ An institutional study enrolling clinically node-negative invasive breast cancer patients $(n=635)$ demonstrated no association between time from radiographic screening (mammography or sonography) to surgery (median 21 days, range 1-132 days) with tumor size or lymph node upstaging. ${ }^{11}$ Similarly, a comparison of serial sonographic images at the time of initial diagnosis 


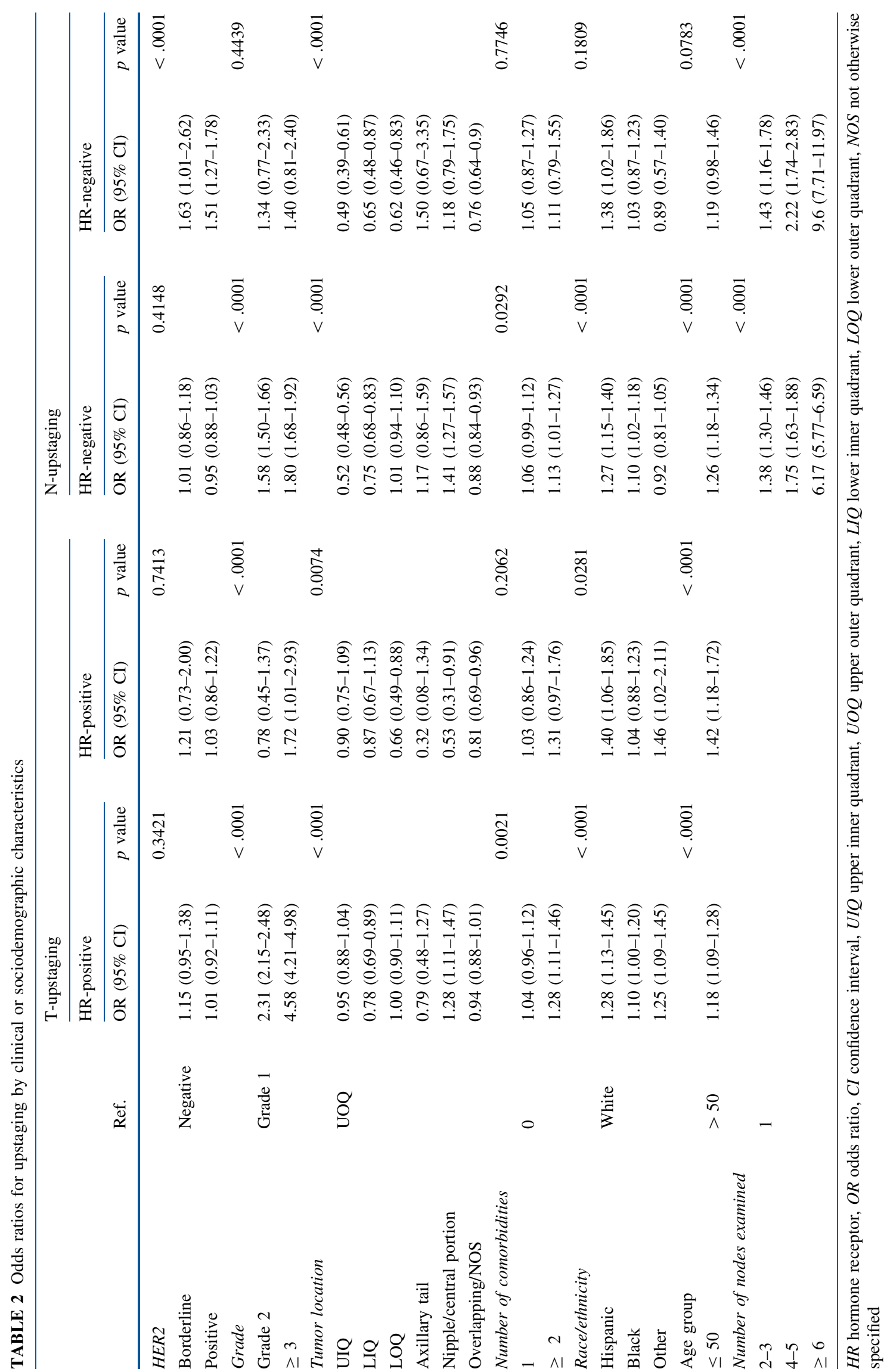


and the day prior to surgery (median 31 , range $8-78$ days) among 323 unifocal invasive breast cancer patients concluded that time between diagnosis and surgery $>30$ days was not associated with tumor growth compared with time $\leq 30$ days. $^{12}$ This discrepancy may be attributable to several factors, including shorter median or range of TTS compared with our cohort (median 28 days in HR-positive and 27 days in HR-negative; range 8-180 days), differing classifications of TTS intervals, and inclusion of a broader range of disease (stage, histology, and HR status). A more recent study for the analysis of cT1-2N0 disease using the NCDB $(n=279,090)$ showed that TTS had no association with upstaging from clinical to pathologic prognostic stage after adjustment; however, a trend of increasing upstaging with prolonged TTS was seen among patients with cT1N0 disease in unadjusted analysis. ${ }^{13}$ One of the key differences in our study is the use of a selective cohort of anatomic cT1N0M0 ductal breast carcinoma patients who received BCS as their first definitive treatment. Since mastectomy is indicated for higher-risk disease (e.g. multifocal, multicentric, family history/genetics, etc.), patients who underwent mastectomy were excluded due to differing disease nature from those who receive BCS. ${ }^{13}$ In fact, mastectomy was a significant predictor of disease upstaging in other studies. ${ }^{8,13}$ Similarly, patients with non-ductal histology such as lobular carcinoma were excluded based on predicted ambiguity of radiographic tumor size measurement reported in lobular carcinoma in situ. ${ }^{13}$ Lastly, our cohort was categorized by TTS intervals of $\leq 30$, $31-60,61-90$, and $\geq 91$ days, with $\leq 30$ days TTS as the reference category based on the observed statistically significant difference in overall mortality observed in each 30-day interval beyond $\leq 30$ days TTS. ${ }^{9}$ Overall, our data highlight the importance of minimizing the preoperative period to avoid the potential risk of disease progression in HR-positive T1N0M0 ductal breast carcinoma.

Similar to upstaging trends reported in DCIS patients, our analysis recapitulated a positive association between upstaging and HER2 positivity, higher histology grade, and younger age in T1N0M0 ductal carcinomas. ${ }^{17-20}$ Our multivariable analysis showed that the nipple/central portion of the breast is significantly associated with increased risk of upstaging relative to tumors located in the upper outer quadrant for HR-positive tumors, presumably due to the convergence of mammary ducts at the nipple-areolar complex. ${ }^{21}$ The high density of fibroglandular tissue in this region results in heightened radiographic opacity that poses a challenge for sensitivity of mammographic detection in retroareolar masses. ${ }^{21}$ Another clinically significant finding of our study was age-related differences in upstaging. This may be attributable in part to the higher prevalence of aggressive subtypes or higher breast density among premenopausal women. ${ }^{19,22}$ Breast Imaging Reporting and
Data System (BI-RADS) category 4 and 5 disease (as opposed to category 3 ) is reported to be consistently and significantly associated with upstaging of DCIS to invasive breast cancer. ${ }^{6}$ Greater breast density, defined by a higher percentage of radiographically dense epithelial and stromal tissue in the breast as opposed to radiographically lucent adipose tissue, both decreases mammographic sensitivity for detecting breast masses and is an independent risk factor for breast cancer regardless of the method by which it is detected. ${ }^{23,24} \mathrm{~A}$ cross-sectional meta-analysis of over 11,000 women found that mammographic density decreased with increasing age in both premenopausal and postmenopausal women, with the greatest reduction in mammographic density occurring at the time of menopausal transition. ${ }^{25}$

During the coronavirus disease 2019 (COVID-19) pandemic, $15 \%$ of cancer patients reported that their inpatient surgical procedures had been affected. ${ }^{26}$ Given the concern over the potentially negative survival impact of excessive surgical delays, the COVID-19 Breast Cancer Consortium published guidelines for operative prioritization. T1N0 estrogen receptor (ER)-positive/HER2-negative patients are categorized into priority level $\mathrm{C} 1$ and recommended neoadjuvant endocrine therapy (NET) in the context of delayed operation ${ }^{27}$ based on the comparable efficacy and overall survival rate of NET to adjuvant endocrine therapy in the GRETA trial. ${ }^{28}$ A NCDB cohort study showed that postmenopausal women with cT2-4c ER-positive breast cancer $(n=2,294)$ treated with NET were 1.6 times more likely to undergo BCS. ${ }^{29}$ Similarly, Minami et al. recently demonstrated that in patients with cT1-2N0 breast cancer, NET use did not impact stage or overall survival, supporting its safe use as a means of delaying surgery in patients with ER-positive breast cancer during the COVID19 pandemic. ${ }^{13}$ Additionally, randomized studies have demonstrated an overall response rate to NET of $>50 \%$ and an NET-induced conversion rate to BCS of $>30 \%$. $^{30-32}$ The duration and type of NET have been suggested to affect the response rate. ${ }^{33}$ While the incremental benefit of NET is evident at a therapy duration of up to 8 months, ${ }^{32,34}$ studies of short treatment duration showed limited efficacy ${ }^{35}$ as the response rate of breast cancer to NET is slow. The P024 trial, during which postmenopausal women with ER-positive stage II-III breast cancers $(n=228)$ underwent treatment with neoadjuvant letrozole or tamoxifen for 4 months prior to surgery, revealed a significantly higher overall response rate in patients treated with letrozole $(55 \%$ vs. $36 \%))^{30,36}$ Despite efficacy in downsizing tumors and the favorable safety profile of NET, ${ }^{31,36}$ the rates of disease progression during NET range from about $5-25 \%,{ }^{30-32}$ partly due to molecular mechanisms that contribute to endocrine resistance. ${ }^{37-40}$ Since the primary focus of randomized trials using NET 
has largely been to downstage clinical stage $\mathrm{T} 2$ or higher tumors, ${ }^{30,34,35}$ its effect on T1N0 breast cancer remains unknown. Since the current study focused on patients who underwent surgery as their primary cancer treatment and excluded NET-treated cases, our data are not generalizable to those who were treated with NET and experienced extended surgical delays during the COVID-19 pandemic. Further follow-up retrospective studies to evaluate the impact of NET on T1N0 HR-positive breast cancer patients using a dataset with capacity for data adjustment for NET responses will be critical in gaining insight into breast cancer management.

The current study has several strengths and limitations. The large cohort size, along with the ability to adjust for multiple tumor properties and demographic characteristics with exclusion of potentially confounding subpopulations, provides, for the first time, a valuable context for understanding of the association between surgical delays and measurable disease progression of both tumor size and nodal status in IDCs. To our surprise, after multivariable analysis, TTS-dependent upstaging remained significant in HR-positive patients, but not in HR-negative patients, even though the likelihood of T-upstaging among HR-negative patients was nearly double that among HR-positive patients $(11.0 \%$ vs. $6.8 \%)$. Additionally, there was no interaction between TTS and HER2 status in any of the models, suggesting both luminal A (HR-positive/HER2-negative) and B (HR-positive/HER2-positive) subtypes are likely to be affected by a delay in surgery. In light of this insight, which seems opposed to current understanding of HR-positive disease as slow-proliferating and HR-negative disease as rapidly-proliferating, ${ }^{41,42}$ it is critical to address the mechanism underlying HR-specific TTS-associated upstaging. One main limitation includes the varying detection sensitivity ${ }^{43-45}$ of radiographic images utilized for clinical staging. Accurate tumor size measurement by mammogram and sonogram remains a challenge, especially in dense breast tissue,${ }^{46,47}$ lobular carcinomas, ${ }^{48}$ and lesions under the nipple/areolar complex. ${ }^{21}$ Since the type of imaging modality and BI-RADS score are unavailable in the NCDB, further investigation with such variables to address the ambiguity of radiographic imaging is necessary to further determine the contribution of TTS to upstaging. Additionally, although we used a clinically selective subpopulation for our analysis, characterized by small earlystage tumors, to substantiate TTS-associated upstaging of invasive breast cancer, this does not negate the possibility of TTS-associated upstaging in higher-stage disease or disease with non-ductal histology.

\section{CONCLUSION}

Overall, preoperative disease progression of clinical T1N0M0 ductal carcinoma patients was largely attributable to intrinsic tumor biology, including subtype, histology grade, and tumor location, and to demographic factors, including age, race/ethnicity, and comorbidities. However, TTS presented a modifiable factor that was significantly associated with both $\mathrm{T}$ - and $\mathrm{N}$-upstaging among HR-positive clinical T1N0M0 ductal carcinoma patients, underscoring the importance of timely surgery.

ACKNOWLEDGMENT Research reported in this publication was supported in part by the National Cancer Institute Cancer Center Support Grant P30CA225520 and the Oklahoma Tobacco Settlement Endowment Trust contract awarded to the University of Oklahoma Stephenson Cancer Center. The content is solely the responsibility of the authors and does not necessarily represent the official views of the National Institutes of Health or the Oklahoma Tobacco Settlement Endowment Trust.

FUNDING This research received no external funding support.

DISCLOSURE Natalie Hills, Macall Leslie, Rachel Davis, Marielle Crowell, Hiroyasu Kameyama, Hallgeir Rui, Inna Chervoneva, William Dooley, and Takemi Tanaka declare no conflicts of interest.

ETHICS APPROVAL AND CONSENT TO PARTICIPATE This was a retrospective cohort study using de-identified data from the NCDB database. Therefore, consent for patient participation and study publication was not required. The study approval was obtained by the University of Oklahoma Institutional Review Board (\#7446).

OPEN ACCESS This article is licensed under a Creative Commons Attribution 4.0 International License, which permits use, sharing, adaptation, distribution and reproduction in any medium or format, as long as you give appropriate credit to the original author(s) and the source, provide a link to the Creative Commons licence, and indicate if changes were made. The images or other third party material in this article are included in the article's Creative Commons licence, unless indicated otherwise in a credit line to the material. If material is not included in the article's Creative Commons licence and your intended use is not permitted by statutory regulation or exceeds the permitted use, you will need to obtain permission directly from the copyright holder. To view a copy of this licence, visit http://creativecommons. org/licenses/by/4.0/.

\section{REFERENCES}

1. American Cancer Society. Breast cancer facts \& figures 2019-2020. Atlanta: American Cancer Society; 2019.

2. Liederbach E, Sisco M, Wang C, Pesce C, Sharpe S, Winchester DJ, et al. Wait times for breast surgical operations, 2003-2011: a report from the National Cancer Data Base. Ann Surg Oncol. 2015;22(3):899-907.

3. Eaglehouse YL, Georg MW, Shriver CD, Zhu K. Time-to-surgery and overall survival after breast cancer diagnosis in a universal health system. Breast Cancer Res Treat. 2019;178(2):441-50. 
4. Smith EC, Ziogas A, Anton-Culver H. Delay in surgical treatment and survival after breast cancer diagnosis in young women by race/ethnicity. JAMA Surg. 2013;148(6):516-23.

5. Glastonbury CM, Mukherji SK, O'Sullivan B, Lydiatt WM. Setting the stage for 2018: how the changes in the American joint committee on cancer/union for international cancer control. AJNR Am J Neuroradiol. 2017;38(12):2231-7.

6. Brennan ME, Turner RM, Ciatto S, Marinovich ML, French JR, Macaskill $\mathrm{P}$, et al. Ductal carcinoma in situ at core-needle biopsy: meta-analysis of underestimation and predictors of invasive breast cancer. Radiology. 2011;260(1):119-28.

7. Bilimoria KY, Ko CY, Tomlinson JS, Stewart AK, Talamonti MS, Hynes DL, et al. Wait times for cancer surgery in the United States: trends and predictors of delays. Ann Surg. 2011;253(4):779-85.

8. Ward WH, DeMora L, Handorf E, Sigurdson ER, Ross EA, Daly JM, et al. Preoperative delays in the treatment of DCIS and the associated incidence of invasive breast cancer. Ann Surg Oncol. 2020;27(2):386-96.

9. Bleicher RJ, Ruth K, Sigurdson ER, Beck JR, Ross E, Wong YN, et al. Time to surgery and breast cancer survival in the United States. JAMA Oncol. 2016;2(3):330-9.

10. Eriksson L, Bergh J, Humphreys K, Wärnberg F, Törnberg S, Czene K. Time from breast cancer diagnosis to therapeutic surgery and breast cancer prognosis: a population-based cohort study. Int J Cancer. 2018;143(5):1093-104.

11. Wagner JL, Warneke CL, Mittendorf EA, Bedrosian I, Babiera GV, Kuerer HM, et al. Delays in primary surgical treatment are not associated with significant tumor size progression in breast cancer patients. Ann Surg. 2011;254(1):119-24.

12. Lee SH, Kim YS, Han W, Ryu HS, Chang JM, Cho N, et al. Tumor growth rate of invasive breast cancers during wait times for surgery assessed by ultrasonography. Medicine (Baltimore). 2016;95(37):e4874.

13. Minami CA, Kantor O, Weiss A, Nakhlis F, King TA, Mittendorf EA. Association between time to operation and pathologic stage in ductal carcinoma in situ and early-stage hormone receptorpositive breast cancer. J Am Coll Surg. 2020;231(4):434-47.

14. American Joint Committee on Cancer. AJCC cancer staging manual. 7th edn. Atlanta: American Joint Committee on Cancer; 2009. p. $347-76$.

15. National Comprehensive Cancer Network. Breast Cancer NCCN evidence blocks (TM). Pennsylvania: National Comprehensive Cancer Network; 2020.

16. Collaborative Stage Data Collection System Manual. Breast, CS Site-Specific Factor 15, HER2: Summary Result of Testing. 2013. http://web2.facs.org/cstage0205/breast/Breast_sbg.html. Accessed 9 Apr 2020.

17. Lee KH, Han JW, Kim EY, Yun JS, Park YL, Park CH. Predictive factors for the presence of invasive components in patients diagnosed with ductal carcinoma in situ based on preoperative biopsy. BMC Cancer. 2019;19(1):1201.

18. Yen TW, Hunt KK, Ross MI, Mirza NQ, Babiera GV, MericBernstam F, et al. Predictors of invasive breast cancer in patients with an initial diagnosis of ductal carcinoma in situ: a guide to selective use of sentinel lymph node biopsy in management of ductal carcinoma in situ. J Am Coll Surg. 2005;200(4):516-26.

19. Mustafa RE, DeStefano LM, Bahng J, Yoon-Flannery K, Fisher CS, Zhang PJ, et al. Evaluating the risk of upstaging HER2positive DCIS to invasive breast cancer. Ann Surg Oncol. 2017;24(10):2999-3003.

20. Holm-Rasmussen EV, Jensen MB, Balslev E, Kroman N, Tvedskov TF. Risk factors of sentinel and non-sentinel lymph node metastases in patients with ductal carcinoma in situ of the breast: a nationwide study. Breast. 2018;42:128-32.
21. Giess CS, Keating DM, Osborne MP, Ng YY, Rosenblatt R. Retroareolar breast carcinoma: clinical, imaging, and histopathologic features. Radiology. 1998;207(3):669-73.

22. Bauer KR, Brown M, Cress RD, Parise CA, Caggiano V. Descriptive analysis of estrogen receptor (ER)-negative, progesterone receptor (PR)-negative, and HER2-negative invasive breast cancer, the so-called triple-negative phenotype: a population-based study from the California cancer Registry. Cancer. 2007;109(9):1721-8.

23. Nazari SS, Mukherjee P. An overview of mammographic density and its association with breast cancer. Breast Cancer. 2018;25(3):259-67.

24. Boyd NF, Guo H, Martin LJ, Sun L, Stone J, Fishell E, et al. Mammographic density and the risk and detection of breast cancer. N Engl J Med. 2007;356(3):227-36.

25. Burton A, Maskarinec G, Perez-Gomez B, Vachon C, Miao H, Lajous $\mathrm{M}$, et al. Mammographic density and ageing: a collaborative pooled analysis of cross-sectional data from 22 countries worldwide. PLoS Med. 2017;14(6):e1002335.

26. American Cancer Society Cancer Action Network. COVID-19 Pandemic ongoing impact on cancer patients and survivors: survey findings summary. Washington: American Cancer Society Cancer Action Network; 2020.

27. Dietz JR, Moran MS, Isakoff SJ, Kurtzman SH, Willey SC, Burstein HJ, et al. Recommendations for prioritization, treatment, and triage of breast cancer patients during the COVID-19 pandemic. the COVID-19 pandemic breast cancer consortium. Breast Cancer Res Treat. 2020;181(3):487-97.

28. Mustacchi G, Ceccherini R, Milani S, Pluchinotta A, De Matteis A, Maiorino L, et al. Tamoxifen alone versus adjuvant tamoxifen for operable breast cancer of the elderly: long-term results of the phase III randomized controlled multicenter GRETA trial. Ann Oncol. 2003;14(3):414-20.

29. Chiba A, Hoskin TL, Heins CN, Hunt KK, Habermann EB, Boughey JC. Trends in neoadjuvant endocrine therapy use and impact on rates of breast conservation in hormone receptor-positive breast cancer: a national cancer data base study. Ann Surg Oncol. 2017;24(2):418-24.

30. Ellis MJ, Ma C. Letrozole in the neoadjuvant setting: the P024 trial. Breast Cancer Res Treat. 2007;105(1):33-43.

31. Smith IE, Dowsett M, Ebbs SR, Dixon JM, Skene A, Blohmer $\mathrm{J}-\mathrm{U}$, et al. Neoadjuvant treatment of postmenopausal breast cancer with anastrozole, tamoxifen, or both in combination: the Immediate Preoperative Anastrozole, Tamoxifen, or Combined with Tamoxifen (IMPACT) multicenter double-blind randomized trial. J Clin Oncol. 2005;23(22):5108-16.

32. Fontein DB, Charehbili A, Nortier JW, Kranenbarg EM-K, Kroep JR, Putter H, et al. Efficacy of six month neoadjuvant endocrine therapy in postmenopausal, hormone receptor-positive breast cancer patients-a phase II trial. Eur $J$ Cancer. 2014;50(13):2190-200.

33. Spring LM, Gupta A, Reynolds KL, Gadd MA, Ellisen LW, Isakoff SJ, et al. Neoadjuvant endocrine therapy for estrogen receptor-positive breast cancer: a systematic review and metaanalysis. JAMA Oncol. 2016;2(11):1477-86.

34. Krainick-Strobel UE, Lichtenegger W, Wallwiener D, Tulusan AH, Jänicke F, Bastert $G$, et al. Neoadjuvant letrozole in postmenopausal estrogen and/or progesterone receptor positive breast cancer: a phase IIb/III trial to investigate optimal duration of preoperative endocrine therapy. BMC Cancer. 2008;8(1):62.

35. LeVasseur N, Willemsma K-A, Li H, Gondara L, Yip WC, Illmann $\mathrm{C}$, et al. Efficacy of neoadjuvant endocrine therapy versus neoadjuvant chemotherapy in ER-positive breast cancer: results from a prospective institutional database. Clin Breast Cancer. 2019;19(6):e683-9. 
36. Eiermann W, Paepke S, Appfelstaedt J, Llombart-Cussac A, Eremin J, Vinholes $J$, et al. Preoperative treatment of postmenopausal breast cancer patients with letrozole: a randomized double-blind multicenter study. Ann Oncol. 2001;12(11):1527-32.

37. Chang M. Tamoxifen resistance in breast cancer. Biomol Ther. 2012;20(3):256.

38. Miller TE, Ghoshal K, Ramaswamy B, Roy S, Datta J, Shapiro CL, et al. MicroRNA-221/222 confers tamoxifen resistance in breast cancer by targeting p27Kip1. $J$ Biol Chem. 2008;283(44):29897-903.

39. Piva M, Domenici G, Iriondo O, Rábano M, Simoes BM, Comaills V, et al. Sox 2 promotes tamoxifen resistance in breast cancer cells. EMBO Mol Med. 2014;6(1):66-79.

40. Ali S, Rasool M, Chaoudhry H, Pushparaj PN, Jha P, Hafiz A, et al. Molecular mechanisms and mode of tamoxifen resistance in breast cancer. Bioinformation. 2016;12(3):135.

41. Raber MN, Barlogie B, Latreille J, Bedrossian C, Fritsche H, Blumenschein G. Ploidy, proliferative activity and estrogen receptor content in human breast cancer. Cytometry. 1982;3(1):36-41.

42. Meyer JS, Prey MU, Babcock DS, McDivitt RW. Breast carcinoma cell kinetics, morphology, stage, and host characteristics. A thymidine labeling study. Lab Investig. 1986;54(1):41-51.

43. Hieken TJ, Harrison J, Herreros J, Velasco JM. Correlating sonography, mammography, and pathology in the assessment of breast cancer size. Am J Surg. 2001;182(4):351-4.
44. Onesti JK, Mangus BE, Helmer SD, Osland JS. Breast cancer tumor size: correlation between magnetic resonance imaging and pathology measurements. Am J Surg. 2008;196(6):844-8 (discussion 9-50).

45. Gruber IV, Rueckert M, Kagan KO, Staebler A, Siegmann KC, Hartkopf A, et al. Measurement of tumour size with mammography, sonography and magnetic resonance imaging as compared to histological tumour size in primary breast cancer. BMC Cancer. 2013;13:328.

46. Kolb TM, Lichy J, Newhouse JH. Comparison of the performance of screening mammography, physical examination, and breast US and evaluation of factors that influence them: an analysis of 27,825 patient evaluations. Radiology. 2002;225(1):165-75.

47. Fletcher SW, Elmore JG. Clinical practice. Mammographic screening for breast cancer. $N$ Engl J Med. 2003;348(17):1672-80.

48. Johnson K, Sarma D, Hwang ES. Lobular breast cancer series: imaging. Breast Cancer Res. 2015;17:94.

Publisher's Note Springer Nature remains neutral with regard to jurisdictional claims in published maps and institutional affiliations. 